\title{
Welding Deformation Prediction of Typical offshore Platform Structures Based on Inherent Strain
}

\author{
Xinyi Yang, Hong Zhou, Lei Wang, Jiancheng Liu, Hongfei Zhang
}

\begin{abstract}
Aiming at the semi-submersible lifting platform, based on the thermo-elastic-plastic finite element analysis, the elastic finite element method is used to predict the welding instability deformation and control the welding deformation of the connecting platform and floating body in the platform. Through the prediction and analysis of welded joints, the inherent deformation is obtained, and then the calculated inherent deformation is loaded into the whole structure in the form of load, and the welding deformation of the whole structure is obtained. Then, by comparing the welding sequence, the scheme of minimum welding deformation is obtained. Then the opening of the structure is compared, and the influence of the opening on the welding deformation of the structure is analyzed. It is used in the welding process of special structure of semi-submersible lifting and disassembly platform to realize the precision control of welding and ensure the welding quality, construction accuracy and strength performance.
\end{abstract}

Index Terms - Welding Deformation; Welding Sequence; Inherent Strain

\section{INTRODUCTION}

With the continuous development of science and technology, modern platforms are developing towards super-large and multi-function[1]. Welding technology is indispensable in the construction of platforms. The structure of the semi-submersible lifting and disassembly platform is special and complex. The special structure of the platform is mostly composed of high strength steel plates, which are mainly connected by welding. As a reliable and efficient joining technology, welding has been widely used in various industrial fields, especially in the construction of ships and marine structures. Compared with other processing technologies, welding technology has the advantages of high production efficiency, good working conditions and high structural strength.

Xinyi Yang Postgraduate of Naval Architecture and Ocean Engineering department of Jiangsu University of Science and Technology, China.+8613655296001

Hong Zhou Professor of Naval Architecture and Ocean Engineering department of Jiangsu University of Science and Technology, China:+8613656136398

Lei Wang Postgraduate of Naval Architecture and Ocean Engineering department of Jiangsu University of Science and Technology, China;+8618260636076China

Jiancheng Liu Merchants (Jiangsu) heavy industry Co., Ltd China. Hongfei Zhang Jiangsu New Yangzi Shipbuilding Ltd, China.

Foundation projects: 2017 Ministry of Industry and Information Technology high-tech ship research project - semi-submersible lifting and disassembly platform development; Postgraduate Research \& Practice Innovation Program of Jiangsu Province (KYCX18_2342)
Welding deformation and stress, as the basic mechanical response, will inevitably occur during and after welding. Welding deformation will have a lot of negative effects on the manufacturing accuracy and strength performance of welded structures. In the actual production process, the welding deformation is usually reduced by post-weld correction, but this method will not only affect the entire construction cycle and cost a lot of manpower and financial resources, but also may cause ${ }^{2}$ damage to welded components. Therefore, the ${ }^{3}$ precise prediction of the welding deformation of typical structures in the platform and the optimization of welding ${ }^{4}$ procedure sequence will have a positive impact on the construction quality and accuracy control of the special structures of the platform.

In recent years, a large number of scholars and experts at home and abroad have focused on the prediction and control methods of welding deformation. UEDA[2] was the first person to apply the theory of inherent strain to the prediction of welding deformation. Three-bar model was used to derive the calculation formula of inherent strain. Ding Zhenbin[3] used inherent strain to predict welding deformation of large and complex hull segments, which was consistent with the measured results, and proved the feasibility of inherent strain method in complex hull structures. Tang Yonggang[4] and others used the inherent strain to predict the surprise welding of the stern section of the hull, and used the predicted results to give the corresponding deformation compensation, which met the requirements of precision control. Liang Wei[5] improved the traditional inherent deformation method and improved the accuracy of predicting welding deformation of thin plate. The connection structure of semi-submersible lifting and disassembly platform is one of the important special structures. In this paper, the welding deformation of semi-submersible lifting and disassembly platform is predicted by numerical simulation using inherent strain, which provides effective basic data for the prediction and control of welding deformation of platform special structure[6].

\section{CALCULATION METHOD}

\section{A. Thermo-elastic Finite Element Method}

In this paper, based on the thermo-elastic-plastic finite element method[7], the typical structure of the platform is analyzed. In the thermal elastoplastic finite element analysis of welded joints, heat conduction and elastoplastic mechanics are mainly considered. Thermal process plays a decisive role 
in the subsequent force process. Therefore, it is necessary to use uncoupled formulas to analyze the thermodynamic behavior in welding process, and then consider the influence of temperature field formed by heat transfer and other physical properties of materials on stress and deformation. Thermo-elastic-plastic finite element method consists of two steps: (1) using heat transfer theory to analyze and calculate the whole transient temperature field; (2) applying the calculated transient temperature distribution as a thermal load to the stress analysis after welding, calculating welding residual stress, plastic strain and displacement.

\section{B. Inherent Stress Theroy}

According to a large number of thermos-elastic-plastic finite element analysis calculation results and experimental observations[8], we believe that the residual stress and welding deformation during welding are caused by the inherent strain $\varepsilon^{*}$, and the inherent strain mainly depends on the type of welded joint and the material property board. Welding parameters such as thickness and heat input. The total strain $\varepsilon^{\text {total }}$ during the heating and cooling cycles of the welding process can be divided into the strain components given by equation (1), namely elastic strain $\varepsilon^{\text {elastic }}$, thermal strain $\varepsilon^{\text {thermal }}$, plastic strain $\varepsilon^{\text {plastic }}$, creep strain $\varepsilon^{\text {creep }}$ and strain produced by phase transformation $\varepsilon^{\text {phase }}$.

$$
\varepsilon^{\text {total }}=\varepsilon^{\text {elastic }}+\varepsilon^{\text {thermal }}+\varepsilon^{\text {plastic }}+\varepsilon^{\text {creep }}+\varepsilon^{\text {phase }}
$$

Furthermore, the total strain can be rearranged as the sum of the elastic strain and the inherent strain $\varepsilon^{*}$, including all strain components except the elastic strain. In other words, the inherent strain $\varepsilon^{*}$ is defined as the sum of plastic strain, thermal strain, and creep strain. The strain caused by the equation (2), especially for carbon steel welded joints, is much less strain due to creep and solid phase transformation, and its inherent strain can be expressed by plastic strain.

$$
\varepsilon-\varepsilon^{\text {elastic }}=\varepsilon^{*}=\varepsilon^{\text {thermal }}+\varepsilon^{\text {plastic }}+\varepsilon^{\text {creep }}+\varepsilon^{\text {phase }}
$$

\section{FinITE ELEMENT MODEL}

\section{A. Material properties}

The typical structure in the semi-submersible lifting and disassembling platform is mainly composed of $12 \mathrm{~mm}$ thick EH36 steel plate. The chemical composition and mechanical properties of EH36 steel plate are shown in Table I and

\begin{tabular}{|c|c|c|c|}
\hline $\begin{array}{c}\text { Chemical } \\
\text { composition }\end{array}$ & $\begin{array}{c}\text { Mass fraction } \\
1 \%\end{array}$ & $\begin{array}{c}\text { Chemical } \\
\text { composition }\end{array}$ & $\begin{array}{c}\text { Mass } \\
\text { fraction } 1 \%\end{array}$ \\
\hline $\mathrm{c}$ & 0.18 & $\mathrm{Cr}$ & 0.20 \\
\hline $\mathrm{Si}$ & 0.50 & $\mathrm{Ni}$ & 0.40 \\
\hline Mn & $0.90 \sim 1.60$ & Mo & 0.08 \\
\hline $\mathrm{P}$ & 0.040 & V & $0.5 \sim 0.10$ \\
\hline
\end{tabular}
Table II.

\begin{tabular}{ccccc}
\multicolumn{3}{c}{ Table II Mechanical properties of EH36 steel plate } \\
\hline \multirow{2}{*}{ Property } & \multicolumn{3}{c}{ Mechanical property } & \multicolumn{2}{c}{$\begin{array}{c}\text { Longitudinal } \\
\text { impact force/J }\end{array}$} \\
\cline { 2 - 5 } & $\mathrm{R}_{\mathrm{eH}}{ }^{\mathrm{a}} / \mathrm{MPa}$ & $\mathrm{R}_{\mathrm{m}}{ }^{\mathrm{a}} / \mathrm{MPa}$ & $\mathrm{A} / \%$ & $-40{ }^{\circ} \mathrm{C}$ \\
\hline $\begin{array}{c}\text { Parameter } \\
\text { values }\end{array}$ & $\geq 355$ & $490 \sim 620$ & $\geq 21$ & $\geq 34$ \\
\hline
\end{tabular}

\section{B. Modeling}

This paper takes the typical structure of offshore platform composed of EH36 high strength steel as the research object. According to the size and composition of the weldment, as shown in figure I, the geometric model of the weldment is established. Considering the calculation accuracy and efficiency comprehensively, to ensure the rationality of mesh generation of the whole welding structure, uniform finite element mesh generation is adopted, all of which use plate and shell elements, the number of nodes is 1488 and the number of elements is 1420 , as shown in figure $\Pi$.

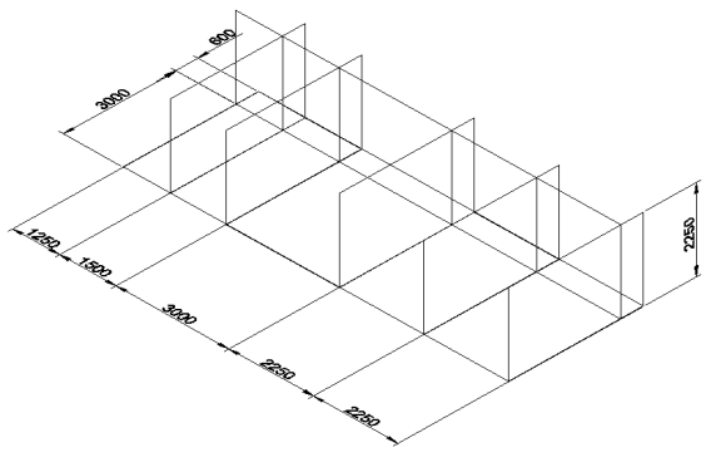

Fig. IGeometric dimensions of Weldments

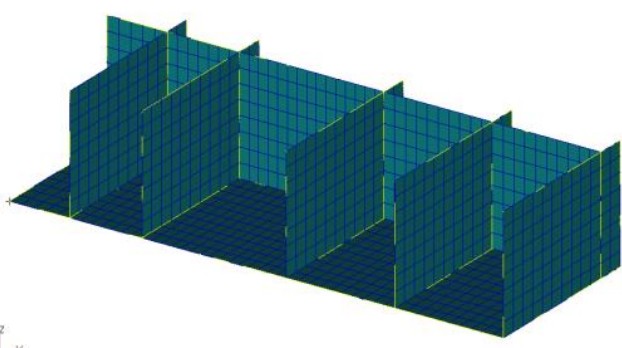

Fig. II Finite element model meshing

\section{CALCULATION RESUltS AND ANALYSIS}

\section{A. Three different welding sequences}

In this paper, the common welding sequence[9] in the actual production process of shipyard is studied. The numbers of the bottom plate and the horizontal and vertical members are shown in the figure III.

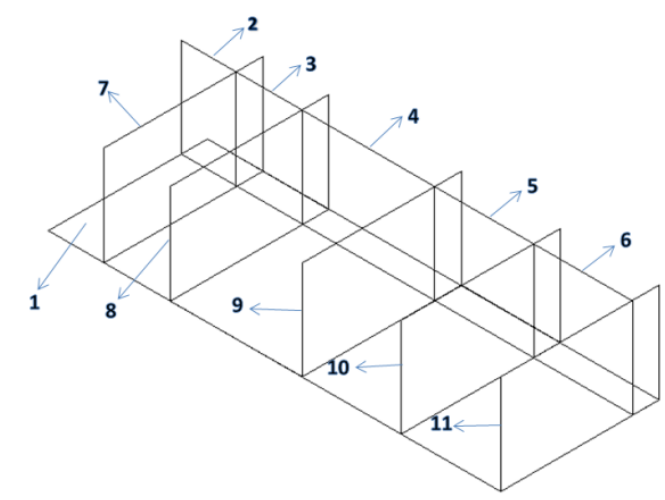

Fig. III Number of bottom plate and the horizontal and vertical members 
The specific welding sequence is shown in Table III .

Scheme 1: With the base plate as the datum, the longitudinal component is welded to the base plate, then the transverse component is welded to the base plate, and finally the connection between the transverse and longitudinal components is completed.

Scheme 2: First complete the connection between the horizontal and vertical components, and then weld the jointed horizontal and vertical components to the bottom plate.

Scheme 3: Take the base plate as the datum, from left to right, complete the connection between the horizontal and vertical components in turn and weld them to the base plate.

Table III Welding sequence schemes for bottom plate and the horizontal and vertical member

\begin{tabular}{ll}
\hline Project number & \multicolumn{1}{c}{ Welding sequence } \\
\hline & (1) $7 、 8 、 9 、 10 、 11 \rightarrow 1$ \\
Scheme 1 & $2 、 3 、 4 、 5 、 6 \rightarrow 1$ \\
& (3) $2 、 3 、 4 、 5 、 6 \rightarrow 7 、 8 、 9 、$ \\
& $10 、 11$ \\
& (1) $2 、 3 、 4 、 5 、 6 \rightarrow 7 、 8 、 9 、$ \\
& $10 、 11$ \\
Scheme 2 & (2) $2 、 3 、 4 、 5 、 6 、 7 、 8 、 9 、$ \\
& $10 、 11 \rightarrow 1$ \\
& (1) $2 \rightarrow 7 \rightarrow 1$ \\
& (2) $3 \rightarrow 7 \rightarrow 8 \rightarrow 1$ \\
& (3) $4 \rightarrow 8 \rightarrow 9 \rightarrow 1$ \\
Scheme 3 & (4) $5 \rightarrow 9 \rightarrow 10 \rightarrow 1$ \\
& (5) $6 \rightarrow 10 \rightarrow 11 \rightarrow 1$ \\
\end{tabular}

According to the above three different welding sequence schemes, the welding deformation distribution reprogram can be obtained by calculating the welding deformation elastic finite element analysis. As shown in figure III the welding deformation clouds of scheme 1, scheme 2 and scheme 3 are presented.

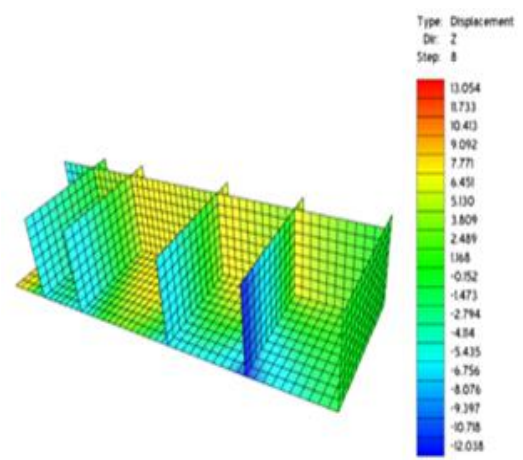

(a) Scheme 1

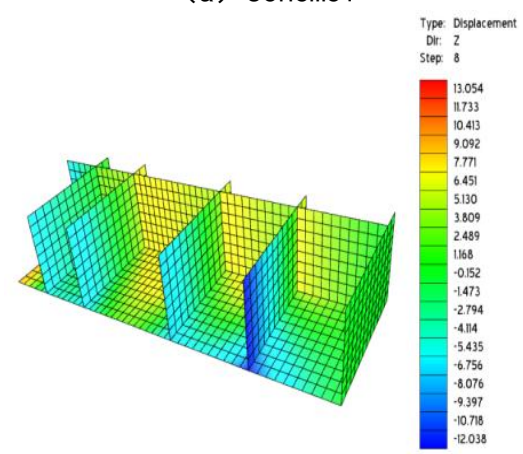

(b) Scheme2

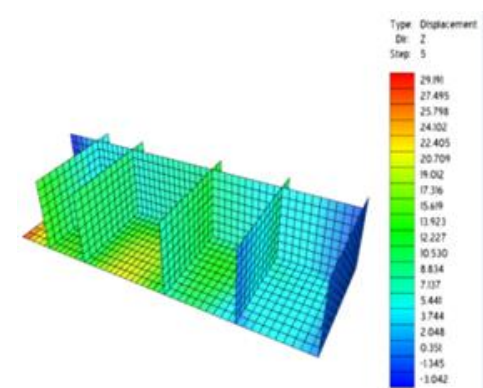

(c) Scheme3

Fig IV Welding Deformation Cloud of Scheme 1-3

In order to observe the deformation of three welding sequences more intuitively, we draw curves from the direction of length and width, and compare them, as shown in figure $\mathrm{V}$. Figure. V (a) is a comparison of welding deformations in the length direction, and figure $\mathrm{V}(\mathrm{b})$ is a comparison of welding deformations in the width direction.

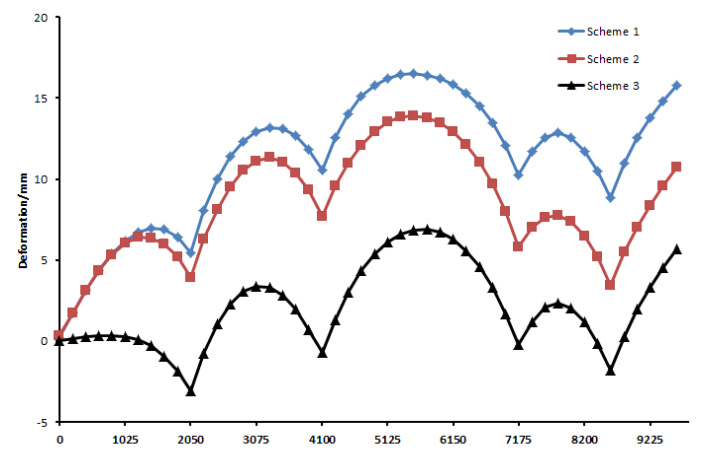

(a) Length

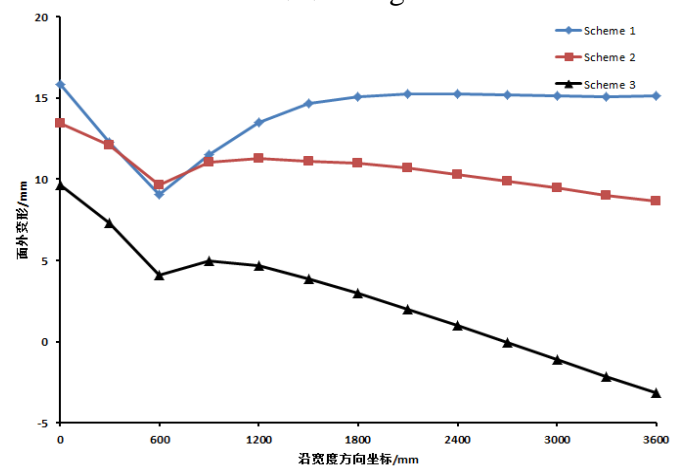

(b) Width

Fig. V Structural deformation in different welding sequence

From the above curves, it can be seen that the deformation trend of the bottom plate after welding is that the middle part is arched upward, and the maximum deformation value in $\mathrm{Z}$ direction appears in the middle part of the bottom plate. In scheme 1 and 2, the welding deformation is larger, the middle part is arched obviously, and the welding deformation obtained in scheme 3 is relatively small, and the deformation range is also smaller.

\section{Effect of Opening on Welding Deformation}

By comparing the above contents, the welding deformation obtained by using the welding sequence of scheme 3 is the smallest. According to the welding sequence of scheme 3, the plate and shell elements are used in the original structure with their own openings. The number of nodes is 1964 and the number of elements is 1838 . As shown in figure. VI, the effect of the openings on the welding deformation of the whole structure is studied. The welding deformation nephogram is shown in figure. VII 

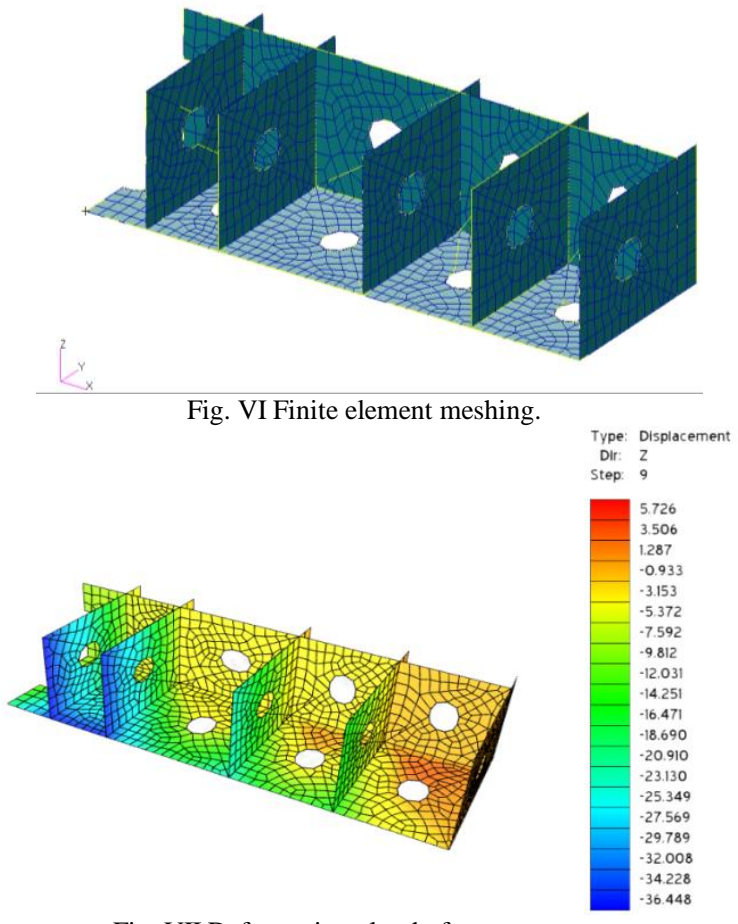

Fig. VII Deformation cloud of open structure

From figure. VII, it can be seen that after considering the opening, the welding deformation of the whole structure increases significantly compared with that of the structure without opening, and the curves of length and width are also compared, as shown in figure. VIII.

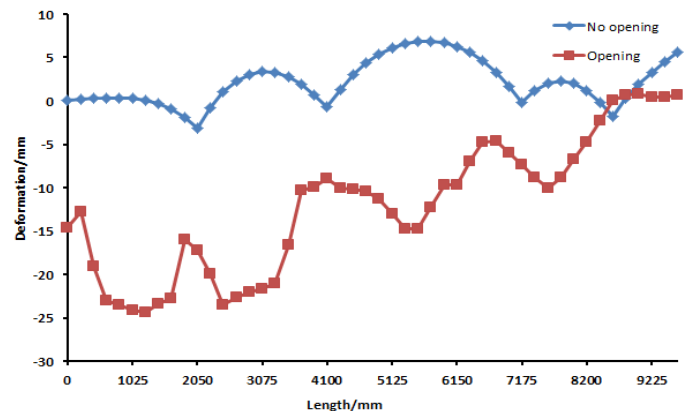

(a) Length

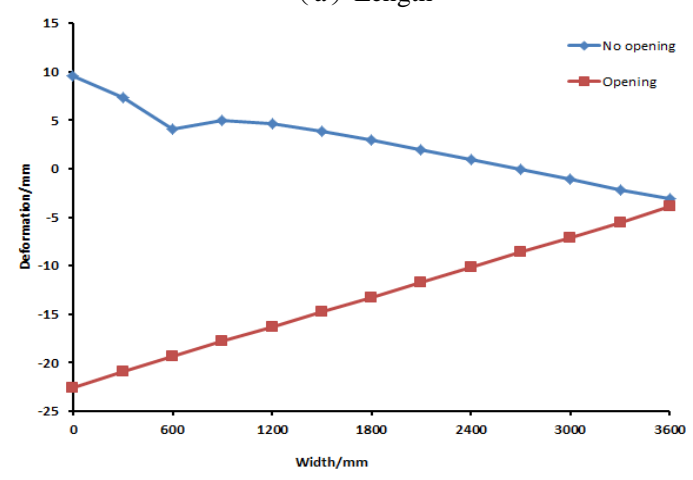

(b) Width

Fig VIII Comparison of Welding Deformations Considering Openings

As can be seen from Fig. 8, considering the opening, not only the welding deformation of the weldment increases, but also the fluctuation of the deformation increases a lot. Therefore, the influence of the opening on the welding deformation of the structure is very important. Therefore, in the calculation of welding deformation, the influence of the opening cannot be ignored.

\section{CONCLUSION}

The structure of semi-submersible lifting and disassembly platform is special and complex. It mainly uses welding technology to complete the connection between components. Therefore, the study of welding deformation is very important for the quality, safety and construction cycle of construction. In this paper, the typical connection structure of the platform is taken as the research object, and some of its structures are selected to predict its welding deformation. The following conclusions are drawn:

(1)The welding sequence has a great influence on the welding deformation of the structure. Through the study of three welding sequences, it is concluded that the welding sequence of scheme 3 has the least influence on the welding deformation.

(2)The opening reduces the stiffness of the plate and increases the welding deformation. Therefore, the influence of the opening on the welding deformation of the structure is very important. In the actual processing process, appropriate measures should be taken to control it.

proper nouns and element symbols. For papers published in translation journals, please give the English citation first, followed by the original foreign-language citation [8].

\section{REFERENCES}

[1] Shu Xinglou. Weldability of heavy plate high strength steel for ship and formation and prevention of welding cracks [J]. Ship, 2009, 20 (2): 9-12.

[2] Ueda Y, Yuan M G, Mochizuki M, etal. Experimental verification of a method for prediction of welding residual stresses in $\mathrm{T}$ joints using inherent strains 4th report: Method for prediction using source of residual stress[J]. Welding International, 1993, 7(11):863-869.

[3] Ding Zhenbin, Zhu Yuanwei, Wang Bo, etal. Segmental welding deformation of large complex hull [J]. China Ship Research, 2011, 06 (3): 79-82.

[4] Tang Yonggang, Chen Qianqing. Research on the accuracy control of finite element calculation of inherent strain in large tail assembly and welding [J]. Shipbuilding Engineering, 2012 (6): 53-55.

[5] Liang Wei, Ma Feng, Zhang Yu, etal. Research on High Precision Prediction Method for Welding Deformation of Thin Plate [J]. Marine Mechanics, 2017 (4).

[6] Xiang Zuquan, Liu Bin, Chen Wentao, etal. Study on welding deformation prediction and control of T-joint of hull structure [J]. Shipbuilding Engineering, 2016 (3): 65-68

[7] Wang Jiangchao, Zhou Hong, Wu Zhengfeng, et al. Progress in computational welding mechanics in ship and marine structure construction [J]. Shipbuilding technology, 2018 (3).

[8] Wang Jiangchao, Zhou Hong, Zhao Hongquan, etal. Comparative study on evaluation of tendon force for welding distortion prediction in thin plate fabrication[J]. China Welding, 2017, 26(3):1-11.

[9] Shi Xionghua, Wang Jiangchao, Xiangsheng, etal. Numerical analysis of the influence of welding sequence on structure deformation of ship hulls [C]. Papers of the 10th Postgraduate Forum on Ships and Marine Engineering in Wuhan. 2017.

[10] Liu Bin. Research on prediction and control methods of angular deformation of T-joint with different grooves [D]. Wuhan University of Technology, 2017.

Xinyi Yang Postgraduate of Naval Architecture and Ocean Engineering department of Jiangsu University of Science and Technology, China.+8613655296001

Hong Zhou Professor of Naval Architecture and Ocean Engineering department of Jiangsu University of Science and Technology, China:+8613656136398

Lei Wang Postgraduate of Naval Architecture and Ocean Engineering department of Jiangsu University of Science and Technology, China;+8618260636076China.

Jiancheng Liu Merchants (Jiangsu) heavy industry Co., Ltd China. Hongfei Zhang Jiangsu New Yangzi Shipbuilding Ltd, China. 